\title{
An let-7 KRAS rs712 polymorphism increases hepatocellular carcinoma risk
}

\author{
D. Xiong ${ }^{1 *}$, Y.P. Song ${ }^{2 *}$, W. Xiong ${ }^{3}$ and Y.D. Liang ${ }^{2}$ \\ 'Department of General Surgery, Meishan People's Hospital, Meishan, \\ Sichuan, China \\ 'Laboratory of Molecular Translational Medicine, \\ West China Institute of Women and Children's Health, \\ Key Laboratory of Obstetric \& Gynecologic and Pediatric Diseases and Birth \\ Defects of Ministry of Education, West China Second University Hospital, \\ Sichuan University, Chengdu, China \\ ${ }^{3}$ Multiple Organ Transplant Center, Sichuan Provincial People's Hospital, \\ Chengdu, China
}

*These authors contributed equally to this study.

Corresponding author: D. Xiong

E-mail: dr_xiongding@163.com

Genet. Mol. Res. 14 (4): 14050-14055 (2015)

Received May 5, 2015

Accepted August 20, 2015

Published October 29, 2015

DOI http://dx.doi.org/10.4238/2015.October.29.24

\begin{abstract}
KRAS, also known as V-Ki-ras2 Kirsten rat sarcoma viral oncogene homolog, acts as an intracellular signal transducer. The oncogenic KRAS mutation is an essential step in the development of many types of human cancers, including hepatocellular carcinoma. Here we aimed to investigate the relationship between KRAS rs712 polymorphisms and hepatocellular carcinoma susceptibility. Five-hundred-and-fourteen participants were enrolled in a case-control study (262 cases and 252 normal subjects). The variants were distinguished using polymerase chain reaction-restriction fragment length polymorphism. Significantly increased $\mathrm{HCC}$ risk was observed to be associated with the T allele of the rs712 locus $(\mathrm{P}=0.049, \mathrm{OR}=1.35,95 \% \mathrm{Cl}=1.01-1.78)$. Further, $\mathrm{HCC}$ risk with the GT genotype $(P=0.015, O R=1.64,95 \% C l=1.08-2.50)$ and the TT genotype
\end{abstract}


$(\mathrm{P}=0.015, \mathrm{OR}=2.56,95 \% \mathrm{Cl}=1.05-6.25)$ in a codominant model was significantly higher than that with the GG genotype. In a dominant model, significantly increased $\mathrm{HCC}$ susceptibility was also associated with T allele carriers $(P=0.006, O R=1.75,95 \% C l=1.16-2.63)$. Moreover, we found that the frequency of the KRAS rs712 TT genotype was significantly higher in HBV-positive HCC patients than in HBV-negative HCC patients.

Key words: KRAS; Hepatocellular carcinoma; Polymorphisms; Genetics

\section{INTRODUCTION}

Hepatocellular carcinoma (HCC) is one of the most common malignancies worldwide and the third leading cause of cancer-related deaths (Jemal et al., 2011). Because of the high fatality rates, the incidence and mortality rates are almost equal. However, half of the new cases and mortalities were estimated to occur in China. The high rates of HCC in China are largely due to the prevalence of chronic hepatitis B virus (HBV) infection (Guo et al., 2007; McGlynn and London, 2011). Despite surgical or locoregional therapies, prognosis remains poor because of high tumor recurrence or tumor progression, and currently there are no well-established effective adjuvant therapies for HCC. The molecular biology of carcinogenesis and tumor progression in HCC has been increasingly understood with intense research in recent years (Owonikoko and Khuri, 2013). Several important intracellular signaling pathways such as the Ras/Raf/mitogen-activated protein kinase/ extracellular-signal-regulated kinase pathway and phosphatidylinositol 3-kinase/Akt/mammalian target of rapamycin pathway have been recognized to be involved in $\mathrm{HCC}$, and the roles of several growth factors and angiogenic factors have also been confirmed (Downward, 2003; McCubrey et al., 2008). However, the molecular carcinogenic mechanism of HCC is still not fully understood.

The V-Ki-ras2 Kirsten rat sarcoma viral oncogene homolog (KRAS) is an effector molecule involved in many signal transduction pathways including mitogen-activated protein kinase, phosphatidylinositol 3-kinase, and Ral guanine nucleotide exchange factor pathways (Dunn et al., 2005; Lim et al., 2005; Lim and Counter, 2005; Castellano et al., 2013). The mutation in the KRAS proto-oncogene is frequently detected in several cancers including HCC (Park et al., 2013a,b; Hou et al., 2014; Jung et al., 2014; Zou et al., 2014). Recently, a genetic variant of rs712 in the 3'untranslated region of the KRAS gene has been reported to be functional in the regulation of KRAS by disrupting the complementary site of let-7 and miR-181 (Kumar et al., 2007; Wang et al., 2012; Kim et al., 2014). The rs712 G allele altered the secondary structure of KRAS and resulted in 15\% derepression in luciferase expression compared to that in the wild-type in HeLa cells (Kim et al., 2014).

Previous studies have revealed that the KRAS rs712 polymorphism is associated with oral squamous cell carcinoma, gastric cancer, and colorectal cancer. To date, no report has been conducted to examine the association between the polymorphism and HCC risk. Therefore, in present study, we aimed to investigate the association between the polymorphism and HCC risk and its clinical features in a Chinese population.

\section{MATERIAL AND METHODS}

\section{Subjects}

The case-control study was performed on genomic DNA extracted from the peripheral 
blood of 262 newly diagnosed HCC patients with ages in the range of 23-88 years (mean \pm SD: $53.26 \pm 12.55$ ) and 252 controls with ages in the range of $17-83$ years (mean \pm SD: $43.35 \pm 12.86$ ) after obtaining informed consent. The HCC patients were consecutively recruited from the Meishan People's Hospital between June 2012 and September 2013. All the subjects recruited were unrelated ethnic Han Chinese. None of the patients had received any medical treatments. Patients suffering from autoimmune hepatitis or toxic hepatitis; primary or secondary biliary cirrhosis or Budd-Chiari syndrome; other tumors except HCC; recurrence of HCC; and liver disease due to parasitosis, diabetes, fatty liver, metabolism disorders, and severe cardiovascular diseases. The diagnosis of these patients was confirmed by a pathological examination combined with positive imaging (magnetic resonance imaging and/or computerized tomography). Controls were cancerfree individuals selected based on data from a community nutritional survey. Written informed consent was obtained from all subjects participating in this study, and the study protocol was approved by the ethics committee of the hospital.

\section{Genotyping}

Genomic DNA of each individual was extracted from $200 \mu \mathrm{L}$ EDTA-anticoagulated peripheral blood samples by a DNA isolation kit from Bioteke (Peking, China). The procedure was performed according to instruction manual. The selected genotypes of rs712 were analyzed using a polymerase chain reaction-restriction fragment length polymorphism (PCR-RFLP) assay. The primers used for amplification were as follows: F: 5'-ATGACAGTGGAAGTTTTTTTTTCCTC-3' and R: 5'-GAATCATCATCAGGAAGCCCAT-3'. The PCR reactions were performed in a total volume of $25 \mu \mathrm{L}$, including $2.5 \mu \mathrm{L}$ 10X PCR buffer, $1.5 \mathrm{mM} \mathrm{MgCl}_{2}, 0.15 \mathrm{mM}$ dNTPs, $0.5 \mu \mathrm{M}$ each primer, 100 ng genomic DNA and $1 \mathrm{U}$ Taq DNA polymerase. The PCR conditions were $94^{\circ} \mathrm{C}$ for 4 min, followed by 32 cycles of $30 \mathrm{~s}$ at $94^{\circ} \mathrm{C}, 30 \mathrm{~s}$ at $60^{\circ} \mathrm{C}$ and $30 \mathrm{~s}$ at $72^{\circ} \mathrm{C}$, with a final elongation at $72^{\circ} \mathrm{C}$ for 10 min. PCR products of the polymorphisms were digested using specific restriction enzyme Taq I for $4 \mathrm{~h}$ at $65^{\circ} \mathrm{C}$ (New England BioLabs Inc; Beverly, MA, USA) and the digested PCR products were separated on a $6 \%$ polyacrylamide gel and stained with $1.0 \mathrm{mg} / \mathrm{mL}$ argent nitrate. About $20 \%$ of the samples were randomly selected for use in the repeated assays and the results were $100 \%$ concordant. The genotypes were confirmed by DNA sequencing analysis.

\section{Statistical analysis}

All data were analyzed using SPSS 13.0 (SPSS Inc, Chicago, IL). Genotype frequencies of rs712 were obtained by direct computing and were evaluated for the Hardy-Weinberg equilibrium by using the chi-square test. Genotypic association tests in a case-control pattern, assuming codominant, dominant, recessive, overdominant, or log-additive genetic models were performed using SNPstats (Sole et al., 2006). Odds ratio (OR) and respective 95\% confidence intervals were determined to evaluate the effects of any difference between alleles and genotypes. $P$ values of 0.05 or less were considered to indicate statistically significant differences between patients with $\mathrm{HCC}$ and the controls.

\section{RESULTS}

rs712 polymorphisms were successfully genotyped in 262 HCC patients and 252 healthy control subjects. Genotype distributions of the polymorphisms in the cases and controls were 
consistent with the Hardy-Weinberg equilibrium. Allele and genotype frequencies are shown in Table 1. Significantly increased HCC risk was observed to be associated with the T allele of the rs712 locus $(\mathrm{P}=0.049, \mathrm{OR}=1.35,95 \% \mathrm{Cl}=1.01-1.78)$. Further, $\mathrm{HCC}$ risk with the GT genotype $(P=0.015, O R=1.64,95 \% C l=1.08-2.50)$ and the TT genotype $(P=0.015, O R=2.56,95 \% C l$ $=1.05-6.25$ ) in a codominant model was significantly higher than that with the GG genotype. In a dominant model, significantly increased HCC susceptibility was also associated with T allele carriers $(P=0.006, O R=1.75,95 \% C l=1.16-2.63)$. Moreover, compared with the $(\mathrm{GG}+\mathrm{TT})$ genotype, the GT genotype was associated with a 1.51-fold higher HCC risk in an overdominant model $(P=0.05, \mathrm{OR}=1.51,95 \% \mathrm{Cl}=1.01-2.27)$.

\begin{tabular}{|c|c|c|c|c|c|}
\hline \multirow[t]{2}{*}{ Genetic model } & \multirow[t]{2}{*}{ Genotype } & \multirow[t]{2}{*}{ HCC patients $\mathrm{N}=262(\%)$} & \multirow[t]{2}{*}{ Controls N = $252(\%)$} & \multicolumn{2}{|c|}{ Logistic regression } \\
\hline & & & & OR $(95 \% \mathrm{Cl})^{\mathrm{a}}$ & $P$ value \\
\hline \multirow[t]{3}{*}{ Codominant } & GG & $150(57.2 \%)$ & $162(64.3 \%)$ & 1.00 & 0.015 \\
\hline & GT & $92(35.1 \%)$ & $79(31.4 \%)$ & $1.64(1.08-2.5)$ & \\
\hline & $\mathrm{TT}$ & $20(7.6 \%)$ & $11(4.4 \%)$ & $2.56(1.05-6.25)$ & \\
\hline \multirow{2}{*}{ Dominant } & GG & $150(57.2 \%)$ & $162(64.3 \%)$ & 1.00 & \\
\hline & GT-TT & $112(42.8 \%)$ & $90(35.7 \%)$ & $1.75(1.16-2.63)$ & 0.006 \\
\hline \multirow{2}{*}{ Recessive } & GG-GT & $242(92.4 \%)$ & $241(95.6 \%)$ & 1.00 & \\
\hline & TT & $20(7.6 \%)$ & $11(4.4 \%)$ & $2.08(0.88-50)$ & 0.14 \\
\hline \multirow[t]{2}{*}{ overdominant } & GG-TT & $170(64.9 \%)$ & $173(68.7 \%)$ & 1.00 & \\
\hline & GT & $92(35.1 \%)$ & $79(31.4 \%)$ & $1.51(1.01-2.27)$ & 0.05 \\
\hline \multirow{4}{*}{ Log-additive } & & & & $1.64(1.63-2.27)$ & 0.0012 \\
\hline & Allele & & & & \\
\hline & $G$ & $392(74.8 \%)$ & $403(79.9 \%)$ & & \\
\hline & $\mathrm{T}$ & $132(25.2 \%)$ & $101(20.1 \%)$ & $1.35(1.01-1.78)$ & 0.049 \\
\hline
\end{tabular}

$\mathrm{N}$ corresponds to the number of individuals. Bold-faced values indicate a significant difference at the $\mathrm{P}<0.05$ level. ${ }^{a}$ Adjusted by age and sex.

To further evaluate whether the variant was associated with certain clinicopathologic features, we performed stratified analyses for genotype distribution and allelic frequency in HCC patients with different characteristics. As shown in Table 2, we found that the frequency of the KRAS rs712 TT genotype was significantly higher in HBV-positive HCC patients than in HBVnegative HCC patients.

\begin{tabular}{|c|c|c|c|c|c|c|c|c|c|c|}
\hline \multirow[t]{2}{*}{ Characteristics } & \multirow[t]{2}{*}{ Total No. $\mathrm{N}=262$} & \multicolumn{3}{|c|}{ Genotype No. (\%) } & \multirow[t]{2}{*}{$P$ value } & \multirow[t]{2}{*}{ OR (95\% Cl) } & \multicolumn{2}{|c|}{ Allele No. (\%) } & \multirow[t]{2}{*}{$P$ value } & \multirow[t]{2}{*}{ OR $(95 \% \mathrm{Cl})$} \\
\hline & & $\overline{G G}$ & GT & $\overline{\mathrm{TT}}$ & & & $\mathrm{G}$ & $\bar{T}$ & & \\
\hline \multicolumn{11}{|c|}{ Smoking status, N (\%) } \\
\hline Smokers & 34 & 22 & 9 & 3 & Ref & & 53 & 15 & 0.65 & $1.22(0.66-2.25)$ \\
\hline Nonsmokers & 228 & 128 & 83 & 17 & 0.51 & $1.20(0.67-2.18)$ & 339 & 117 & & \\
\hline \multicolumn{11}{|c|}{ Drinking status, $\mathrm{N}(\%)$} \\
\hline Drinker & 211 & 120 & 76 & 15 & Ref & & 316 & 106 & 0.44 & $1.28(0.72-2.30)$ \\
\hline Nondrinker & 51 & 30 & 16 & 5 & 0.72 & $1.33(0.45-3.96)$ & 44 & 19 & & \\
\hline \multicolumn{11}{|c|}{ Tumor stages, N (\%) } \\
\hline$I+I I$ & 76 & 44 & 26 & 6 & Ref & & 114 & 38 & 1.00 & $1.01(0.66-1.57)$ \\
\hline ш & 194 & 106 & 66 & 14 & 0.98 & $1.05(0.59-1.87)$ & 278 & 94 & & \\
\hline \multicolumn{11}{|l|}{ HbsAg, N (\%) } \\
\hline Positive & 128 & 74 & 38 & 16 & Ref & & 186 & 70 & 0.27 & $0.80(0.54-1.19)$ \\
\hline Negative & 134 & 76 & 54 & 4 & 0.005 & $4.16(1.32-12.5)$ & 206 & 62 & & \\
\hline
\end{tabular}

$\mathrm{N}$ corresponds to the number of individuals. Bold-faced values indicate a significant difference at the $\mathrm{P}<0.05$ level. 


\section{DISCUSSION}

In this study, we investigated the association between the let-7 KRAS rs712 polymorphism and the risk of $\mathrm{HCC}$ in a Chinese population. Ours is the first study to report an association between the KRAS rs712 polymorphism and a significantly increased risk of HCC.

$K R A S$, also known as V-Ki-ras2 Kirsten rat sarcoma viral oncogene homolog, acts as an intracellular signal transducer. The oncogenic KRAS mutation is an essential step in the development of many types of human cancers including HCC (Lujambio and Lowe, 2012; Hou et al., 2014). In recent years, miRNAs have been recognized as key regulators of cancer development, progression, and metastasis. The role of let-7, one of the first miRNAs to be discovered, has been extensively studied in almost all types of cancers. In addition, the expression of let-7b, -7g, -7i, -7d, -7a, -7c and $-7 \mathrm{eg} / \mathrm{l}$ was found to be lower in HCC cells than in normal hepatocytes, and overexpression of let-7g/i significantly inhibited cell proliferation and promoted cell apoptosis (Shimizu et al., 2010), thus suggesting that the let-7 miRNA may be involved in the growth of HCC cells.

The human KRAS 3'UTR contains multiple putative let-7 complementary sites (LCS), which enables let-7-mediated regulation of KRAS activity. KRAS has been reported to be negatively regulated by the let-7 miRNA family. The KRAS LCS6 SNP has been intensively investigated as it disrupts the let-7 miRNA binding site, resulting in increased expression of KRAS (Chin et al., 2008). Chin et al. (2008) were the first to report that the KRAS LCS6 variant allele was significantly associated with an increased risk of developing NSCLC among moderate smokers. Subsequently, the KRAS LCS6 variant allele was reported to confer an increased risk for breast cancer (Hollestelle et al., 2011) and triple negative breast cancer in families with the BRCA1 mutation (Paranjape et al., 2011), but not for invasive epithelial ovarian cancer (Pharoah et al., 2011). In this study, we detected the KRAS LCS6 variant in a Chinese population; however, no polymorphism was observed. Consequently, we focused on the KRAS LCS1 (rs712) SNP, which was observed to influence the susceptibility to gastric cancer, oral squamous cell carcinoma, and colorectal cancer.

Overall, our study has demonstrated that the KRAS rs712 polymorphism was associated with an increased risk of $\mathrm{HCC}$ and was related to the clinical features of the disease, suggesting that KRAS is a risk factor for HCC. Although the precise molecular mechanism remains unknown, we hypothesize the TT genotype may disrupt the let-7 binding site, leading to the abnormal expression of KRAS, which would finally increase susceptibility to HCC. There were still limitations in our study. One is the molecular mechanism is not involved in the study. Another is that our sample size is limited, further research focused on the different areas or populations should be carried out to verify our findings.

\section{Conflicts of interest}

None of the contributing authors have any conflict of interest, including specific financial interests of relationships and affiliations relevant to the subject matter or materials discussed in the manuscript.

\section{ACKNOWLEDGMENTS}

We want to thank Dr. Chen Peng for technical and statistical assistance. This work was supported by grants from Meishan People's Hospital. 


\section{REFERENCES}

Castellano E, Sheridan C, Thin MZ, Nye E, et al. (2013). Requirement for interaction of PI3-kinase p110alpha with RAS in lung tumor maintenance. Cancer Cell 24: 617-630.

Chin LJ, Ratner E, Leng S, Zhai R, et al. (2008). A SNP in a let-7 microRNA complementary site in the KRAS 3' untranslated region increases non-small cell lung cancer risk. Cancer Res. 68: 8535-8540.

Downward J (2003). Targeting RAS signalling pathways in cancer therapy. Nat. Rev. Cancer 3: 11-22.

Dunn KL, Espino PS, Drobic B, He S, et al. (2005). The Ras-MAPK signal transduction pathway, cancer and chromatin remodeling. Biochem. Cell Biol. 83: 1-14.

Guo H, Zhou T, Jiang D, Cuconati A, et al. (2007). Regulation of hepatitis B virus replication by the phosphatidylinositol 3-kinase-akt signal transduction pathway. J. Virol. 81: 10072-10080.

Hollestelle A, Pelletier C, Hooning M, Crepin E, et al. (2011). Prevalence of the variant allele rs61764370 T>G in the 3'UTR of KRAS among Dutch BRCA1, BRCA2 and non-BRCA1/BRCA2 breast cancer families. Breast Cancer Res. Treat. 128: 79-84.

Hou W, Liu J, Chen P, Wang H, et al. (2014). Mutation analysis of key genes in RAS/RAF and PI3K/PTEN pathways in Chinese patients with hepatocellular carcinoma. Oncol. Lett. 8: 1249-1254.

Jemal A, Bray F, Center MM, Ferlay J, et al. (2011). Global cancer statistics. CA Cancer J. Clin. 61: 69-90.

Jung CK, Little MP, Lubin JH, Brenner AV, et al. (2014). The increase in thyroid cancer incidence during the last four decades is accompanied by a high frequency of BRAF mutations and a sharp increase in RAS mutations. J. Clin. Endocrinol. Metab. 99: E276-285.

Kim M, Chen X, Chin LJ, Paranjape T, et al. (2014). Extensive sequence variation in the 3' untranslated region of the KRAS gene in lung and ovarian cancer cases. Cell Cycle 13: 1030-1040.

Kumar MS, Lu J, Mercer KL, Golub TR, et al. (2007). Impaired microRNA processing enhances cellular transformation and tumorigenesis. Nat. Genet. 39: 673-677.

Lim KH and Counter CM (2005). Reduction in the requirement of oncogenic Ras signaling to activation of PI3K/AKT pathway during tumor maintenance. Cancer Cell 8: 381-392.

Lim KH, Baines AT, Fiordalisi JJ, Shipitsin M, et al. (2005). Activation of RalA is critical for Ras-induced tumorigenesis of human cells. Cancer Cell 7: 533-545.

Lujambio A and Lowe SW (2012). The microcosmos of cancer. Nature 482: 347-355.

McCubrey JA, Steelman LS, Abrams SL, Bertrand FE, et al. (2008). Targeting survival cascades induced by activation of Ras/ Raf/MEK/ERK, PI3K/PTEN/Akt/mTOR and Jak/STAT pathways for effective leukemia therapy. Leukemia 22: 708-722.

McGlynn KA and London WT (2011). The global epidemiology of hepatocellular carcinoma: present and future. Clin. Liver Dis. 15: 223-243, vii-x.

Owonikoko TK and Khuri FR (2013). Targeting the PI3K/AKT/mTOR pathway: biomarkers of success and tribulation. Am. Soc. Clin. Oncol. Educ. Book. 2013. Doi: 10.1200/EdBook_AM.2013.33.e395.

Paranjape T, Heneghan H, Lindner R, Keane FK, et al. (2011). A 3'-untranslated region KRAS variant and triple-negative breast cancer: a case-control and genetic analysis. Lancet Oncol. 12: 377-386.

Park JY, Kim WY, Hwang TS, Lee SS, et al. (2013a). BRAF and RAS mutations in follicular variants of papillary thyroid carcinoma. Endocr. Pathol. 24: 69-76.

Park SJ, Sun JY, Hong K, Kwak JY, et al. (2013b). Application of BRAF, NRAS, KRAS mutations as markers for the detection of papillary thyroid cancer from FNAB specimens by pyrosequencing analysis. Clin. Chem. Lab. Med. 51: 1673-1680.

Pharoah PD, Palmieri RT, Ramus SJ, Gayther SA, et al. (2011). The role of KRAS rs61764370 in invasive epithelial ovarian cancer: implications for clinical testing. Clin. Cancer Res. 17: 3742-3750.

Shimizu S, Takehara T, Hikita H, Kodama T, et al. (2010). The let-7 family of microRNAs inhibits Bcl-xL expression and potentiates sorafenib-induced apoptosis in human hepatocellular carcinoma. J. Hepatol. 52: 698-704.

Sole X, Guino E, Valls J, Iniesta R, et al. (2006). SNPStats: a web tool for the analysis of association studies. Bioinformatics 22: 1928-1929.

Wang XF, Shi ZM, Wang XR, Cao L, et al. (2012). MiR-181d acts as a tumor suppressor in glioma by targeting K-ras and Bcl-2. J. Cancer Res. Clin. Oncol. 138: 573-584.

Zou M, Baitei EY, Alzahrani AS, BinHumaid FS, et al. (2014). Concomitant RAS, RET/PTC, or BRAF mutations in advanced stage of papillary thyroid carcinoma. Thyroid 24: 1256-1266. 